\title{
EL GERENTE Y LA CULTURA DE LAS EMPRESAS EN EL PARAGUAY*
}

\section{Consuelo García de Benavides}

D urante los últimos 30 afios, cn Estados Unidos, Europa y Japón, numcrosos autores han estudiado con interés las organizaciones, su gestión, su dinámica, los hombres que la componen y la cultura que ellos engendran. Sin cmbargo, estc auge no se produjo en América Latina, donde cstos temas se han tratado cscasa y marginalmente. Todavia se ignora lo que sucede en esta parte del continente respecto do la gerencia, sus estilos y la cultura de las empresas.

Por ello, el objetivo del presente cstudio es abordar esta realidad a partir del caso específico de las empresas del Paraguay, país que constituye un caso singular en relación al resto de Amćrica Latina, y al cual los trascendentes acontecimientos ocurridos en los últimos ticmpos en todos los campos de suquchacer convierten en un

- Tesis para optar la Maestría en Administración y Gestión en la Universidad Católica de Lovaina, 1991. interesante objeto de estudio. En efecto, cl Paraguay es un país meditcrránco, prácticamente el único país latinoamericano bilingüe en sentido estricto, vivió un proceso de colonización no violento y durante casi todo su periodo republicano estuvo regido por dictaduras, la última de las cuales tcrminó hace muy pocos afos.

\section{Marco de análisis}

La investigación parte de una hipótesis de basc scgún la cual cl sistcma social influye significativamente sobre las cmpresas y los hombresque trabajan en ellas, configurando un cstilodegestión y una cultura propios de esas empresas y con los cuales se forma su identidad.

El marco conceptual utilizado se ha construido a partir de los fundamentos tcóricos de un grupo de autores.

En primer lugar se considera el aporte de. I nhmann ouicn ha innovado las cien- 
cias sociales al introducir la teoría de la evolución. Su esquema permite trabajar las relaciones y las diferencias. Según su enfoque, las organizaciones sociales desarrollan ciertos mecanismos deacción como resultadode un proceso de autorreferencia, de autopoiesis: la variación, la selección y la estabilización. Así, este marco tcórico incluye la probabilidad y la posibilidad como factores importantes para cl análisis de la socicdad.

Luhmann propone tres diferenies niveles de análisis del sistema social (Figura 1). cada uno de los cuales contiene al anterior: cl macrosistema, que corresponde al ámbito mundial; el sistema nacional propio de cada país; y el microsocial, el de las empresas, resultado de la interacción dinámica de los dos anteriores.

etnia nacional. Se estima que cstas variables son significativas y pueden explicar las diferencias entre un país y otro.

La ctnia se manifiesta en los gestos, los comportamientos, las instituciones, las acciones y las obras, así como en las reglas generadoras de un significado común.

Dentrodel componentecultural se ubica el ctnotipo, cuya función es la de la autoconservación del sistema. Trata de ascgurar el éxito del filtro que ejerce sobre los inputs, de proteger al sistema contra las fuerzas que vienen del medio ambiente y producen el stress.

El rol de filtro cn el sistema político es cjercido por los grupos de presion; son ellos los que seleccionan a la entrada del sistema, la información, las demandas $y$ las iniciativas de todo tipo.

Para comprender los comportamicntos que tienen lugar en los medios sociales así como en los grupos de esa socicdad y poder visualizar lactnia nacional, se analizalosarquetipos de Jung, presentes en el sistema

El presente estudio cubre los dos últimos niveles de análisis del sistema. Para estudiar el nivel nacional se sigue a Michaud, para quien la sociedad tiene cuatro componentes: cconómico, político, social y cultural, $y$ un clemento central: la síquico det inconsciente colectivo de toda sociedad.

Finalmente, el nivel microsocial, el análisis de la cultura creada por las cmpresas se basa en los planteamientos de Shein. 
Thevenet y Morgan, autores que han aportado una nueva dimensión al estudio de la cultura en la empresa. A diferencia de otros estudiosos, ellos no consideran que la cmpresa tenga una cultura, sino que la empresa en sí misma es una cultura.

Todo este marco de análisis permite visualizar tres elementos: empresa, cultura y gerente, los cuales se influyen recíprocamente creando la dinámica necesaria para construir y crear la acción organizada de su funcionamicnto. Estos elementos hacen posible la reificación de la cmpresa a través de sus estructuras, sus reglamentos y sus mecanismos de acción. Por otro lado, producen la innovación que todas las cmpresas necesitan para adaptarse a los cambios constantes. El interés del trabajo está en observar y determinar cómo se realiza esta sinergia.

La empresa se concibe como la representación de una parte de larealidad, creada y sostenida socialmente por los miembros que la componen. Lacultura es una manera do construir esa realidad creada socialmente c intcriorizada por sus miembros para darle un significado a su acción. El gerente es el actor simbólico que tiene por función primaria favorecer, promover y desarrollar los modelos descables de orientación administrativa, significativa y đe liderazgo. La administración simbólica es compartir la vida organizacional de manera tal que la acción coordinada de la empresa aumente.

Dado que los fenómenos que se reconocen como medio ambiente son extensión de las propias concepeiones, la cultura existe en el seno de la sociedad y de la organización cmpresarial y ayuda a reinterpretar la naturaleza y el significado de la relación entre la organización y el medio ambiente. De otro parte, la cultura, que depende de ideologías y de los valores que sostienen su accionar, contribuye a la comprensión đel cambio en la empresa. No se trata solamente de cambios tocnológicos, sino también de actitudes y motivaciones de sus miembros. El cambio cultural pone el acento más sobre las personas que sobre las técnicas del cambio.

Se ha tomado como clemento de anílisisal gerente. Sedeterninarí cómo influye sobre la organización y las personas que forman parte de clla. Este punto se basa teóricamente en los trabajos de Mintziberg. que ayudan a comprender mejor el rol del gerente cn la empresa y en la configuración de su cultura.

Es en su rol de líder que el gerente debe poner en prictica la maneracomo larealidad debe ser construida; de alli, la importancia de poner en evidencia las dos caras de una misma moneda. Por un lado, un gerente con liđcrazgo asume el que una situación sea desarrollada a partir de los miembros de la empresa y estimula lo esencial del sistema emergente de signifieación cultural. De esta manera puede integraradecuadamente los intereses de la cmpresa y los de sus miembros. Por otro lado, el líder puede generar una ausencia de valores y la exclusión de ideologías como símbolo de manipulación del poder y de la cultura. La utilización de la manipulación idcológica y el control de la estrategia de administración producen la disociación en lugar de facilitar la expresión de caracteres humalnos sanos en la empresa.

Todo cllo encuentra explicación en el inconsciente colectivo, que constituye la base de los comportamientos nacionales. Las manifestaciones positivas del arquetipo-unificador y sano- causa la integra- 
ción de la cmpresa; mientras que las manifestaciones negativas del mismo arquetipo -manipulador, disociador y dominantepueden terminar por desintegrar a los miembros de la organización.

Las aproximaciones globales integran así en la gestión estratégica los factores culturales y de comportamiento, partiendo del principio según el cual la relación de cada individuo con la organización pasa por una cierta "representación". Esta representación influye fuertemente sobre el funcionamiento de la empresa.

Es nececario entonces ser conscientes de todos los mecanismos existentes en la organización, y poner particular atención en la necesidad de contar con un gerente eapaz de conducir a la empresa bajo valores positivos y principios morales y éti$\cos$ que contribuyan a mejorar cl nivel y la ealidad de vida de la sociedad.

\section{Metodología}

El estudio, de carácter exploratorio, sc realizó con base en tres elementos de observación: entrevistas en profundidad; entrevistas de opinión; y la historia del Paraguay, incluida la dimensión mítica presente en cuentos, mitos y leycndis. Obviamente, los dos primeros requirieron la preparación de instrumentos ad-hoc y la obtención de dos muestras distintas.

Las entrevistas en profundidad se aplicaron a una mucstra (A) de miembros del primer nivel de dirección de sus organizaciones - gerentes generales, presidentes de directorio-, mientras que el cuestionario se aplicó a una muestra (B) de trabajadores de empresas privadas de diferentes tamaños y sectores. Con ambos instrumentos se buscaha básicamente lo mismo: determinar las características culturales de las cmpresas. Se exploró la comunicación, el clima organizacional y cl cstilo de gestión. El interés cstaba en poder responder a las siguientes interrogantes:

a) ¿Cuál es la manera de organizar y de motivar y el estilo de gestión de los directivos en las empresas paraguayas?

b) ¿Los estikos prevalecientes se expliean por las "teorías universales" que sc enscñan en las universidades y escuclas dc administración?

c) ¿Cuáles son las características de la cultura de las empresas y cuál cs la relaciór que existe entre ella, la cultura del país , los gerentes como elementos predominantes en las empresas?

d) iExisten realmente diferencias er los aspectos considerados por este trabajc entre las empresas paraguayas? Si las hay ¿cuáles son?

\section{Análisis de la información y resulta dos}

\subsection{La cultura nacional}

El Paraguay posce una realidad socia propia que lo caracteriza y lo diferencia do otras naciones. En clla, dos culturas se hat influenciado recíprocamente: la aboriger y la espanola. Esta última predomina fren te a otras que también cstán presentes en lt etnia paraguaya, pero que no la han marca do tanto. La cultura española es la de ma yor significación en la formación de la raíces étnicas, arquetipales, políticas : culturales del Paraguay. Está en los cuatri cjes del sistcma social: cconómico, cultu ral, politico y social; los cuales se analizat a continuación. 


\section{El sistema económico}

Paraguay presenta una estructura económica débilmente desarrollada y esencialmentc agraria. La estructura de su producto bruto interno (PBI) por sectores (Figura 2) revela que el Estado genera, administra y controla la mayor parte de la actividad económica.

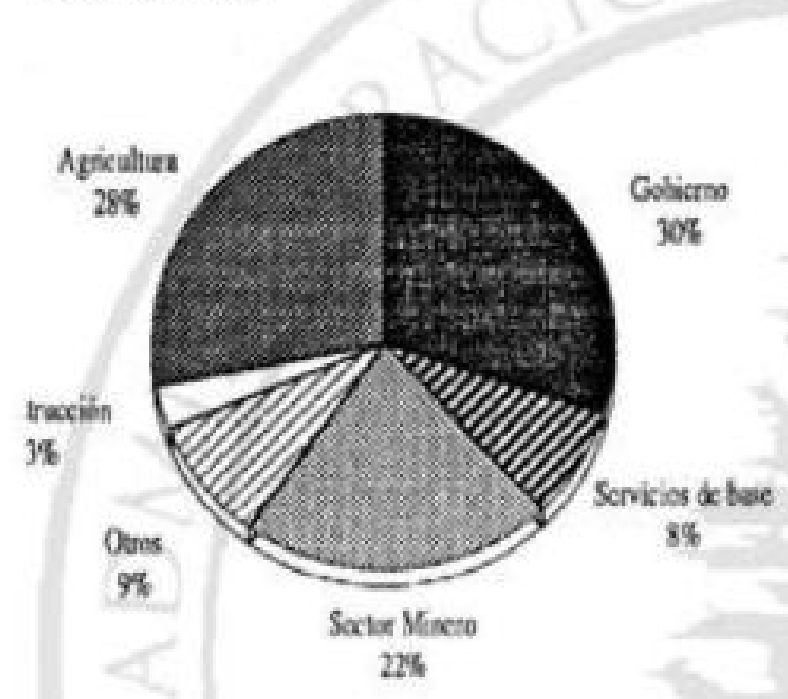

\section{Paraguay: Producto Bruto Interno (estructura porcentual)}

Figura 2

Los recursos naturales del Paraguay hoy en día son la tierra y la fuerza de trabajo. El desequilibrio social proviene esencialmente de la existencia de grandes latifundios y una oligarquía enriquecida por cl comercio y el contrabando que se opone a la agricultura de subsistencia de los minifundios". La forma de organización de la oconomía se basa principalmente en el modelo español de acumulación de riqueza, propiedades y dincro.

1) Encyclopedia Universalis, France S.A., Editeura Paris, 1990, Corpus 17. Aunque al gunos lo consideren paradójico, la infonnación de esta fuente es mucho más objetiva que la proporcionada por historiadores paraguayos.
Los recursos para el desarrollo industrial-incipiente-sc orientan hacia el agro, concretamente hacia actividades agroindustriales, dado el interés de los inversionistas por los resultados obtenidos con productos como cl algodón.

La mayoria de las empresas son comcrciales, de servicios o financieras. En el Cuadro I se les ha clasificado de acuerdo a su tamaño -definido por el número de trabajadores que emplean-y a su forma de propicdad. Como pucde observarse, la mayoría de las empresas son pequeñas y de propicdad privada.

El sistema cultural: lengua, educación, religión

Elsistema cultural puede ser descrito como un conjunto a la vez cstático y dínámico que actủa como un circuito cerradoy cuyos principales elementos están conectados en un juego de ciclos e interaceiones de interinfluencia, donde la ctnia constituye el pivot. Se caractcriza por el intereanbio de mensajes, por una doble producción de información: la propia del mismo sistema y la producida en respuesta a la que viene del extcrior.

En el campo de la lengua existe un amplio rango que va desde el solamente español hasta cl solamente guaraní, pasando por diversos grados del "yopara", mezcla de las dos lenguas. Por ello es dificil afirmar quc hay un bilingüismo claro en el Paraguay ${ }^{2}$, como sí to hay en otros países. Aqui dos lenguas coexisten, se mezclan y se influencian mutuamente.

2f Economia 90, Faculhud de Ciencias Economicas, Administrativas y Contables, Universidad Nacional de Asunción, 6 (6), San L.orenzo C.U., 1990. 


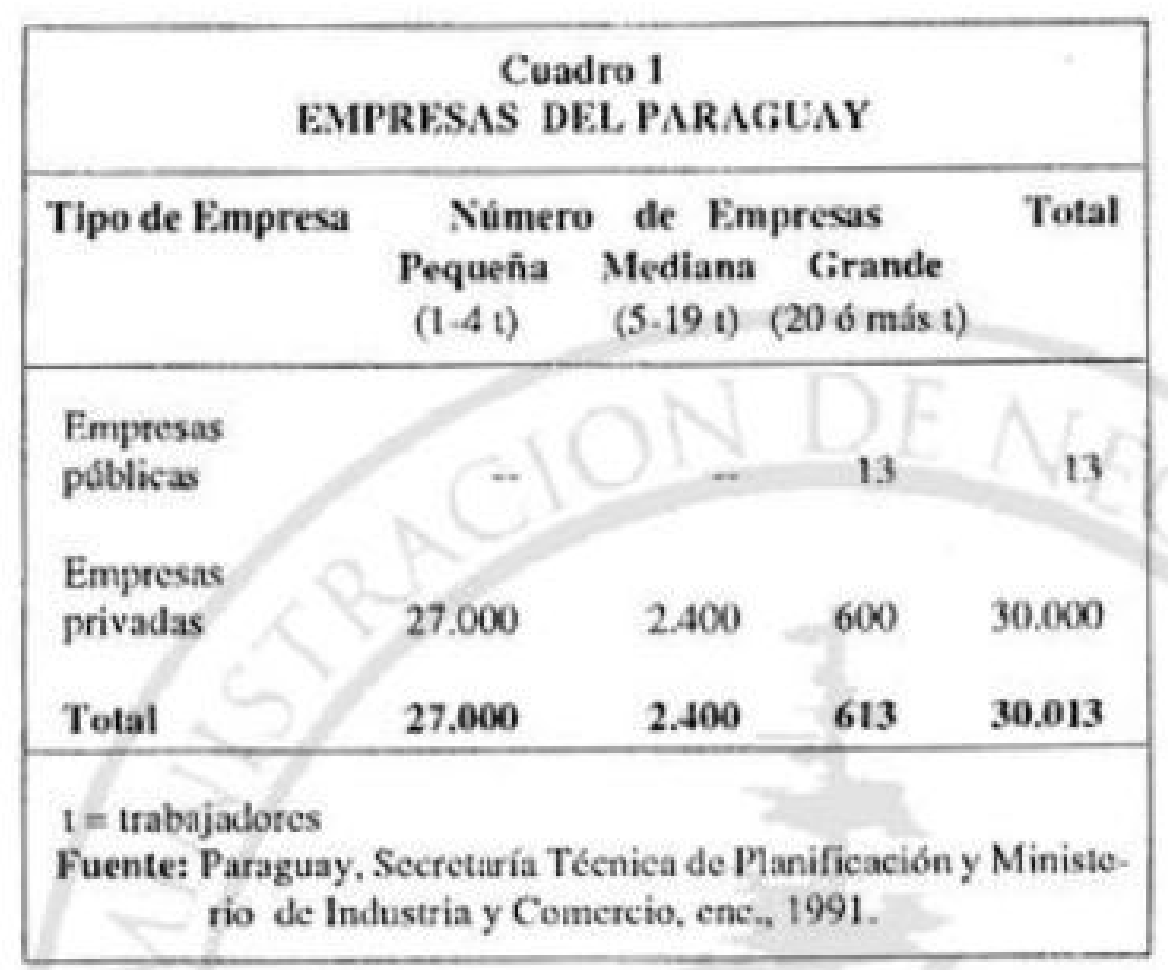

fértil c infantil, cuya actitud "inocente" hizo posible que los valores cristianos se transmitieran y se impregnaran en todos los niveles de la socicdad.

La religión ha sido. pucs, la primera fuente de programación mental utilizada por quienes gobernaron $\mathrm{c}$ influyeron para crear las bases de la socicdad paraguaya: $y$ csta función se ha mantenido a lo largo de la historia de este país.
Aunque la Constitución del país establece que deben utilizarse ambas lenguas, la cducación formal y las comunicaciones públicas se dan en español; el guarani está considerado, más bien, como una lengua secundaria. A pesar de cllo, la lengua autóxtona está presente en las relaciones informales de todos los niveles de la sociedad.

La educación formal considera tres niveles: primaria, secundaria y universitaria, y según una ley que data de 1909, la cducación primaria es obligatoria y gratuita.

En cuanto a la religion, este factor ha sido sicmpre un punto neurálgico $\mathrm{en} \mathrm{cl}$ Paraguay, pues ha influido de manera determinante en todos los componentes de la sociedad. Como se sabe, la cvangelización fue el primer instrumento de transculturizacion utilizado por los espafioles. Como el concepto de Dios existía entre los nativos, fue muy fácil para los conquista* dores introducir la religión católica. Los misioneros encontraron un pucblo dócil,
La ingerencia de la iglesia católica, y de la orden jesuita en especial, en los asuntos del Estado ha sido muy significativa desde la época de la colonia. Aun en la actualidad el poder de la iglesia sigue vigente. Por cjemplo, la religión católica es la religión oficial del país y la Constitución establece que el presidente debe profesar esta fe. Además, durante la dictadura de Suroessner se utilizó la religión como elemento manipulador en oposición al comunismo.

\section{E.tnia}

Los guaranies, a difereneia de las grandes civilizaciones amcricanas como la Inca o la Azteca, no llegaron a alcanzar un gran desarrollo cultural. El caricter scmisedentario de los dos grandes grupos de tribus que poblaron el Paraguay no lo permitió. Sin cmbargo, hasta nucstros días la agricultura subsiste como modelo de organización de su decarrollo sociocconómico, obviamente con la influencia de los estilos españoles. 
Las alianzas familiares siguen manteniendo hasta hoy su rol de base del comportamicnto del pueblo paraguayo. Sc ics encuentra en los cuatro cjes de la sociedad. Así, no es extraño encontrar muchos paricntes ocupando cargos públicos; igualmente, aún es frecuente el matrimonio entre micmbros de familias "prestantes" para reunir las fortunas más importantes; y en cl campo cmpresarial, es común que los micmbros de la familia ocupen los puestos cjecutivos y de dirección. Una manera de "hacer carrera en la cmpresa" es casarse con la hija o el hijo del dueño.

\section{EI sistema politico: Derecho, poder, normas}

La politica en forma organizada llegó al Paraguay con los cspañoles. Al principio las mujeres aborigenes se unicron a los conquistadores a travês de alianzas matrimoniales, cumplicndo con el "tovarazgo", el pacto cntre losdos pucblos. Los guaranies concibicron las alianzas familiares con españoles como un medio de protección: los conquistadores los defendían de otras tribus gucrreras. Por su parte, para los espafoles constituian una forma de asegurar su descendencia y, sobre todo, de lograr la fácil sumisión de la población aborigen.

Este esquema de comportamiento de unión familiar para asegurar el poder so puede obscrvar hasta nucstros días. Las ideologias totalitarias y represivas, la corrupción y las dictaduras sucesivas han marcado prof undamente la socicdad y sus mecanismos de acción en general.

Se tendrá que esperar a las nuevas generaciones para que los resultados de la democracia actúcn de mancra positiva, a través de una integración sicológica de bases profundas. Será necesario buscar en las raíces sicosociológicas del pucblo, los elementos que sirvan de soporte y fijación auténtica y sana de una nucva programación mental en la vida de los paraguayos.

\section{El sistema social: comunicación}

Como ya se ha mencionado, a pesar del status oficial del espaniol, los paraguayos sc comunican cn guarani. La lengua aborigen cs la más importante de las herencias históricas de este pucblo. Los paraguayos suclen decir que solamente en guaraní se pucden expresar los sentimicntos más profundos y en la forma más bella. Un claro cjemplo de esto es la poesfa guarant. También la Biblia fue traducida a cste idioma.

Por otro lado, cn el cje de lo social se asignan y transmiten los roles en la socicdad. Así, por cjemplo, la mujer adcuicre un rol protector y de sumisión basado eu la imagen religiosa de la Virgen Maria, madre y refugio ante sufrimicntos. En el origen cstá cl machismo, que ha sido reforzado a lo largo del tiempo en toxtas las socicdades latinoancricanas.

El resultado del análisis de los cuatro cjes de la sociedad paraguaya se pucde observar gráficamente en el Diagrama 1.

\section{gerente}

3.2 La cultura de la empresa y el

La información recogida y sistematizada a partir de las entrevistas en profundidad se analizó utilizando $\mathrm{cl}$ modclo conceptual presentado $y$ se puede resumir en $\mathrm{cl}$ Diagrama 2.

\section{Aspectos generales}

Se pudo observar que la mayoría $(Y)$ por ciento) de los directivos de las cmpresas 


\section{CARACTERISTICAS DE IA SOCIEDAD PARAGUAYA}

Madre naturaleza, fuente de

vida (arq̧octipo positive).
Poder protostor del viejo sabio, utilisado por los dictadores (respresentación arquetipal negativa).

Potritico

\section{BCONONHCO}

EINLA

"Tdentidad"

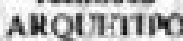

Manufestaciones

Viejo sabie(Dies único, cristiano) Espeilod.

(Nonde nu Teavede, guasand) Guaran!

Transformación (vida ctema, cristiano) Español

(Simbolo del 7, guarani) Guataní

La gran Made (la Virgen María, cristiano) Espatol

Kerana, la naturaleza, guaranDGuaraní

SOCLI

La imagen de ta mujer como refugio ante el sufriasiento $y$ de sumisión (arquetipo negativo).

Principio del machismo.

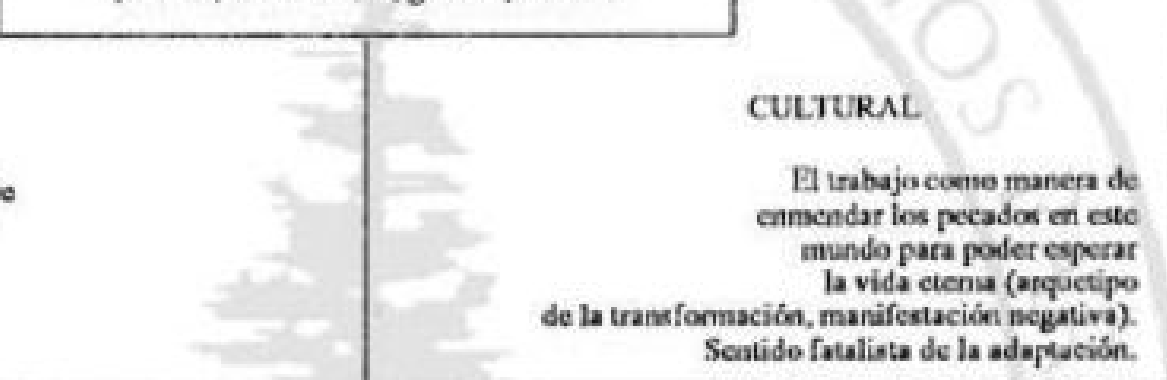

\section{BASES DE LA IDENTIDAD PARAGUAYA}

\section{ECONOMICO}

Estructura econónica dihil. Recursos naturales rios. Modelo de comportanaicuto de "prebendas", paivilegies y compeión.

\section{SOCTAL}

Coeasmacación doble.

Coecepro de roles.

\section{rot.ritico}

Poder absolutista de lenta demoeratixación. Partidox politions emon una idcología tokalitaria durante numerusos ailes.

Nomas legales cumo instromento de cambio.

\section{CULTURAL.}

Lengea bilingüe y "yopura". Edecacién inapropiada para las necesidades de la realidad nacional.

Religión católica fuentemonie enriyada a nivel nacienal. 


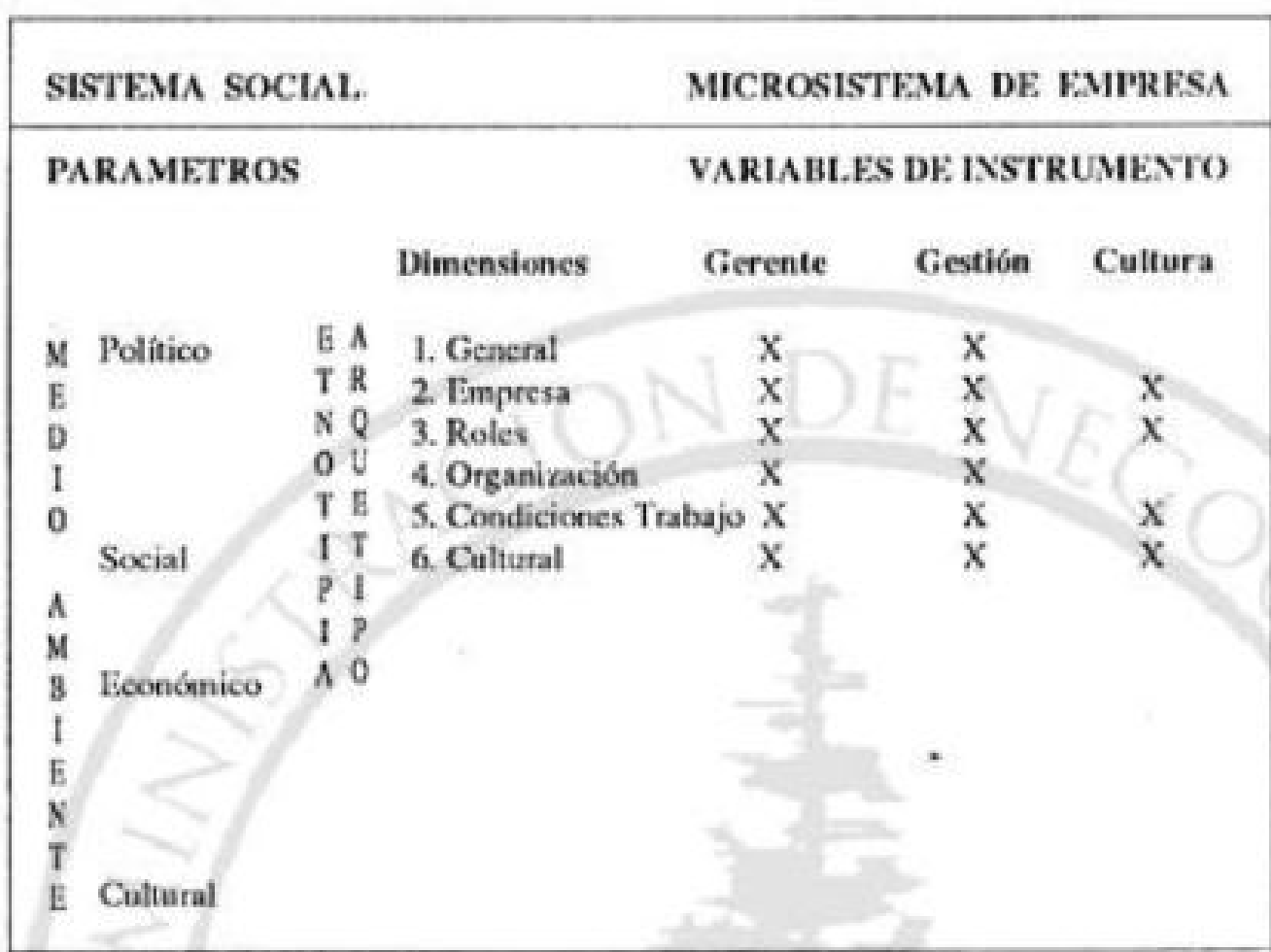

Diagrama 2

Falleto ${ }^{3}$ : "Es necesarioque no se confunda la socicdad anónimadel Paraguay comparíndola con la de países desarrollados, pues aquí cs un pequeño grupo de dirigentes el que concentra el control de un gran número de empresas". De los entrevistados, la mayor parte estaba a la cabcza de entre cuatro y cinco empresas.

son también sus propietarios. Ellos intervienen directamente en la gestión transmitiendo un estilo tradicional y familiar que se caracteriza por la intuición y "cl olfato" para el negocio, con predominio del voluntarismo personal. Respecto de la empresa, mancjan un horizonte de corto plazo.

Son en su totalidad de sexo masculino y preparan a los hijos varones para hacerse cargo de la cmpresa. La mayoría ha dedicado cntre 25 y 30 años a la cmpresa, y la cdad en que el trabajo se transforma en ocupación fluctúa alrededor de los 25 años de cdad. El nivel educativo alcanzado es, en general, cl superior, con frecuencia obtenido en el cxtranjero (Argentina y Uruguay, mayormente).

\section{La empresa}

El origen de las cmpresas continúa siendo esencialmente familiar. Como scñala

\section{E.1 gerente}

El análisis está basado en los cuatro roles del gerente según Mintzberg (Mintzberg 1990). En el Paraguay, el gerente constituye un símbolo que sirve de nexo entre el interior y el cxtcrior de la empresi en cl cual ésta interactúa. En el interior es considerado como el padre-líder que resuelve Ios problemas y como el filtro cntre la empresa y su medio ambiente.

El gerente centraliza toda la información a la cual tiene acceso a través de sus vínculos sociales y dadas las prerrogativas y privilcgios que le otorgan su ubicación social, y la difunde o no de acucrdo a su propio criterio. De csta manera refucrza su posición como centro de poder y consolida

3/ E. Falleto, "Fil empresario industrial en Paraguay", Revista Paraguaya de Sociologia, 2(4). 1965. 
un manejo de dominación de las incertidumbres internas y extcrnas a la empresa, el cual utiliza como argumento de negociación, obteniendo ciertas ventajas.

Sin embargo, toma decisiones no con base en la información que posee, sino en la cxperiencia ganada y por intuición; y según su parecer, rara vez se equivoca.

Ha consagrado a la empresa casi toda la vida -"csta es mi obra y yo debo velar por ella"-, La mayor parte de su ticmpo en ta empresa lo dedica a la negociación externa. Al interior de la empresa, la política de "puertas abiertas" le permite conocer por canales informales - las personas de confianza- todo lo que cn ella succde.

De lo antes dicho se infiere que en los gerentes pucile haher una fijación de las manifestaciones arquetipales del gran principio, representado por Tupa, símbolo de fortaleza, y que se expresa por $\mathrm{cl}$ control absoluto y el paternalismo basados en aspectos carismáticos. Estaactitud aparentemente positiva de sobreproteger al personal equivale a la actitud del padre que debe afrontar todos los problemas porque sus hijos aún no cstán preparados para ello.

\section{Las características organizacionales}

La poca conciencia del gerente de las estructuras políticas, cconómicas, sociales y culturales se hace evidente en su empresa, donde no existe una estructura organizacional definida ni reglas claras y conocidas por todos. Esto se puede interpretar como un reforzamiento de la imagen de omnipotencia del gerente, de ser él de quiendependen todos los acontecimientos.

En el Paraguiay, los gerentes poseen la ideologia "del jefe", propia de su estilo eminentemente tradicional. Se movilizan a través de moxlelos de vigor, pero todavía con una débil aspiración al cambio.

\section{Las condiciones de trabajo}

Son las consideradas por la legislación vigente en loque concierne a remuneraciones (sucldo mínimo más clevado de Sudamérica), horario (de acuerdo con las condiciones climatológicas y costumbres, se hace un alto al medio día para almorzar en casa y hacer siesta) y vacaciones (generalmente todos las toman cn un mismo mes, verano de preferencia). No existen normas explícitas internamente, to cual permite al gerenie determinar cualquier incentivo en función a su percepción de "buen trabajador".

\section{La cultura}

De acuerdo a la pereepeión del gerente, la cultura de la cmpresa es "buena", existe un clima de confianza y sus miembros se relacionan entre sí como "cn familia". Los trabajadores son como una prolongación de sus parientes consanguíncos; por este motivo se espera de cllos respeto, confianza y fidelidad.

Esta cultura parecicra responder a dos vertientes. Por un lado, la todavía reciente dictadlura que đuró 35 años consolidó cn la socicdad la figura del lider carismático con una idcología patcrnalista. Por otro lado, la base arquetipal guaraní percibe este tipode manifestación protectora como un símbolo divino de paternalismo auténtico.

Esta cultura se caracteriza por:

Vulores. Fidelidad, scricdad, responsabilidad, la búsqueda de la calidad en lo que cada uno emprende, respeto por los 
principios cristianos, ambiente de fraternidad y camaradería.

Mitos. Los mitos de la creación y la transformación son fundamentales. Hay una evidente necesidad de cambio, pero los paraguayos creen que éste no depende de ellos; lo perciben como algo exterior a elios.

Slmbolos. El signo predominante está en la lengua guaraní, medio de comunicación por excelencia en términos de confianza y confidencialidad. Los gerentes conocen la lengua, pero evitan usirla porque es considerada como lengua del pueblo. Otro símbolo es la imagen del Fundador, ideal y ejemplo a seguir.

Ritos. El rito más importante es la ceremonia y celebración del aniversario de la empresa. Otro es el cotidiano ritual del "Terere". A todo nivel, mientras se trabaja o se hace un alto en la labor, se comparte el consumo de la yerba mate. Por último, están las fiestas religiosas, todas las cuales han sido incorporadas del medio social. cultural y político del país. Cada empresa tiene su santo protector.

Héroes. Estos son representados por el Fundador, de quien se desprenden los patrones de comportamiento. Otra figura de este tipo pero de carácter religioso cs, por ejemplo, la Virgen Maria, protectora contra todo mal.

Sc ha podidoconstatar que la culturaen las empresas del Paraguay refleja la cultura de su sociedad. De acuerdoa la perspectiva cognitiva, los gerentes de las empresas poseen los indices del saber y de la experiencia que determinan los hechos del medio. Deciden, analizan y cstablecen modelos de comportamicnto propios que se introducen como reglas al ámbito de la empresa y que revelan su lógica de acción consciente.

También se ha encontrado que la motivación, el liderazgo y la estructura de la organización están fucrtemente ligadas a la cultura predominante en el país. Así, se pueden mencionar algunos valores que expresan la filosofia empresarial: respeto por el cliente, como expresión de la utilidad colectiva; excelencia profesional, como la estimación del esfuerzo y la perseverancia; deber de los propietarios, como manifestación de la integración.

\subsection{La cultura de la empresa y los} trabajadores

La aplicación đel cuestionario de opinión permitió levantar la información necesaria para inferir cómo los trabajadores perciben la cultura de la empresa paraguaya. Se les pidió que evaluaran un conjuntode 25 fítem aplicables a su empresa, bajo dos circunstancias diferentes: a) la situación actual, "¿cómo es su empresa en este momento?" y b) la situación ideal, "¿cómo le gustaria que fuera su empresa?". En la Figura 3 se representan las dos serics de calificaciones, una de lo real y otra de lo esperado.

Losresultados obtenidos muestran que, en términos generales, la realidad percibida por los trabajadores y sus expectativas son coincidentes.

La mayor parte de los individuos tienen muchas expectativas por conocer la misión y objetivos de la empresa $y$, aunque los conocen sólo parcialmente, su percepción de la empresa es buena. La lealtad hacia la institución donde trabajan es alta; consideran que la empresa conoce lo que ellos esperan de ella. Se piensa que la empresa 


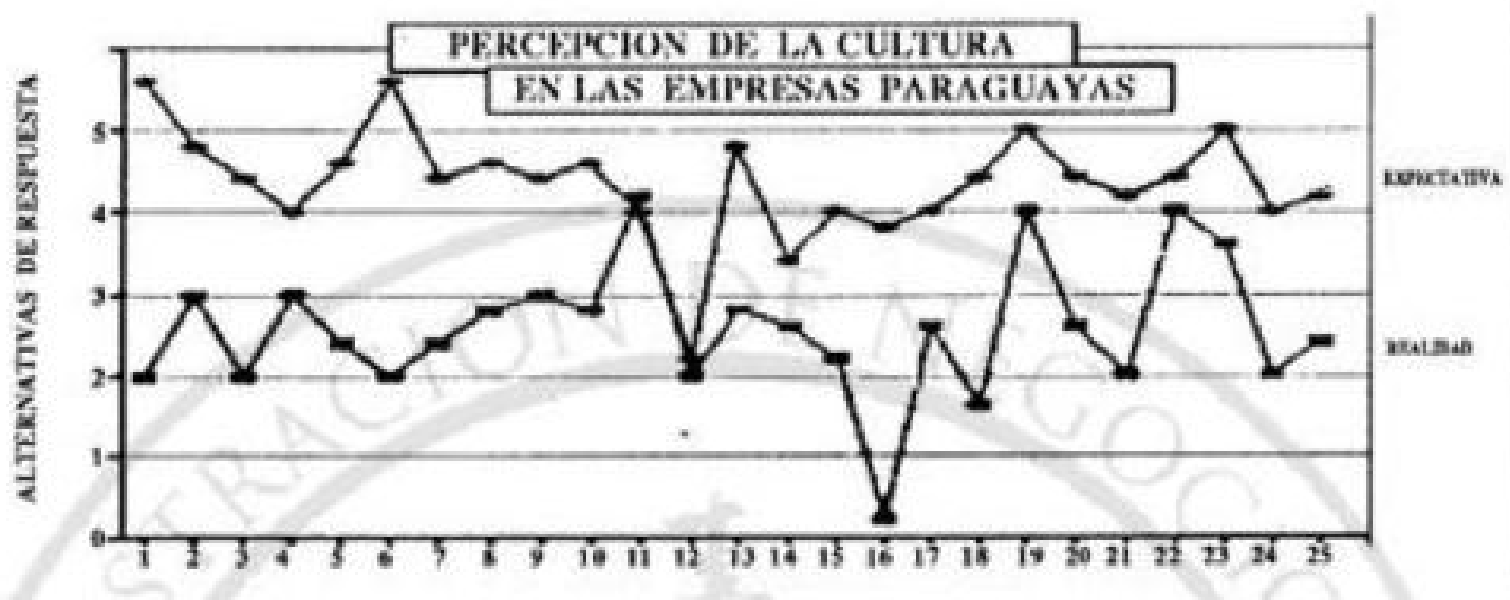

Item Evaluados

1. Misión y objetivas de la cmp̨resa

2. Percopeién de la emprcss

3. Retroalimentución de los objetives expendos

4. Lealtad

5. Cunocimiento de ta cmprusa do las necosidades de sus trabajadores

6. Coherenela entre el discurso de los directives $y$ los hechos

7. Preocupación de la empresa por rotener a sus urabajadenes

8. Cirado de innovación

9. Lógicas de administración prodeciblus

10. Apoyo al tider

11. Recompensas pot iniciativas creativas

12. Calidad de las comunicacimes imemas

13. Trate a las pecsonas
14. Condicianes de trabajo

15. Apotte de los trabajadores a su emipress

16. Participaciōns de ks trabujadores en las decisiones

17. Trabajo on grupos

18. Litimulos

19. Bquipos de trabajo

20. Remuneraciones

21. Recompensa por apoyo de los trahajadorex

22. Desamelle prefesinnal

23. Dasamelle persenal

24. Desarrolle de carrea dentro de la empresa

25. Cohereacia de valeses con las acciones realizadas

Figura 3

no hace esfuerzos para retener a los trabajadores, pero el trabajador ticne altas cxpectativas al respecto. También es alta su expectativa por innovaciones en el discurso, aunque más bien son conservadores. La lógica del gerente es aceptada fácilmente por los trabajadores $y$ hay un apoyo incondicional al líder en la organización. La calidad de la comunicación es percibida como aceptable y así mismo cl tratamiento a las personas. Creen tener buenas condiciones de trabajo y reconocimiento por su aporte, pero no se sienten estimulados. Los valores en relación a las acciones realizadas son considerados coherentes con las lógicas de acción de la cmpresa y los principios que las movilizan.

Sin cmbargo, es preciso destacar que las expectativas se distancian mucho de la realidad en dos ítem. Hay una diferencia notable en el caso de la coherencia entre el discurso y los hechos de los directivos, y en el caso de la participación de los trabajadores en las decisiones de la cmpresa. Es decir, se detecta un aspecto manipulatorio 
en los directivos y un gran interés de los trabajadores por tener un rol más activo en el desenvolvimiento de la empresa. Se estaria, pues, ante la presencia de elementos generadores de cambios futuros en las cmpresas paraguayas.

También se pidió a los trabajadores que cvaluaran de mancra general el clima social en la empresa. En una escala del 0 al 10. la calificación promedio fue 8, lo que indicaría un ambiente de trabajo positivo.

\section{Conclusiones}

El presente trabajonos ha permitidoprobar la validez, del modelo teórico desarrollado para abordar el estudio de la empresa paraguaya. $\mathrm{Se}$ ha cncontrado que:

a) Enel Paraguay intcractúan tres niveles sociales. El primero de ellos, el macrosocial, está constituido por los países del mundo y por las naciones vecinas. Estas últimas forman su medio ambientc más próximo y companten algunos aspectos de su historia, manifestaciones arquetipales, lengua, costumbres, dificultades y tipo de desarrollo. El segundo nivel es el de su propia realidad social, distinta y particular, con características propias en los campos económico, social, político y cultural, y con un elemento central: la ctnotipia, que integra los cuatro ejes y les permite definir el sistema de significación de la sociedad. Finalmente, el nivel microsocial está constituido por las organizaciones empresariaIes, que comparten esta realidad particular y reflejan las características de la sociedad paraguaya; lo que las hace diferentes de cualquier otra cmpresa del mundo. Esto prucba nuestra hipotesis de base.

b) El Paraguay es un país poseedor de una identidad propia que lo diferencia de otros países. Esta afirmación se sustenta cn el estudio de su historia, que muestra cl camino recorrido por este pucblo a través del tiemp̣o, $y$ en el análisis de sus mitos y Icyendas, que permitió detcrminar las manifestaciones arquetipales del inconsciente colectivo de csta sociedad, donde existe un Ienguaje de significación y una comunicación con códigos propios.

c) De esta mancra, el tomar contacto con su realidad social y sus códigos de significación de modo integral permitió abordar las organizaciones empresariales y observarlas bajo una perspectiva amplia y de mayor profundidad, otorgando especial atención a la cultura que en cllas se origina, se comparte y se vive de manera dinámica.

d) El estudio de caso realizado en las empresas del Paraguay permitió reunir todas las variables del modelo al aplicar la metodología planeada en el trabajo de campo. Como resultado, encontramos que las cmpresas y la sociedad paraguayas son parte de una misma realidad, donde tiene lugar una acción permanente de mutua influencia, ya que sus miembros perienecen a ambos contextos y forman parte también de diferentes grupos de esta anplia realidad.

e) Se ha encontrado también que las características de la sociedad del Paraguay cjercen una profunda influencia sobre aspectos relevantes del seno de las empresas, especialmente sobre su cultura, que busca los mecanismos para afrontar el contexto. superar la ansiedad que ello origina y encontrar el equilibrio entre las fuerzas internas y externas de la organización, dada la pujanza de las estructuras $\mathrm{c}$ influencias de dominación que hasta hace muy pocose dejaban sentir en el eseenario social. 
f) Se ha encontradoque en las empresas del Paraguay predominan los estilos tradicionales de gestión, en los cuales los propietarios constituyen la cabeza visible de la organización y el filtro entre la sociedad y su microsociedad. De los gerentes entrevistados, el 80 por ciento era propietario de entre cuatro y cinco empresas de diferentes sectores, predominando las comerciales.

g) El paternalismo en las empresas -tanto públicas como privadas- es una constante. En este aspecto se funden la historia nacional y el impacto causado por los sistemas políticos dictatoriales, los cuales han hecho de las personas simples instrumentosal servicio de sus objetivosy. como consecuencia, desarrollado en ellas una actitud pasiva y una conducta sumisa ante los hechos en la empresa.

h) Todos los hallazgos mencionados a lo largo del trabajo inducen a pensar que la programación mental de la población del Paraguay gira alrededor del scllo profundo causado por los acontecimientos históri$\cos$. Así, ya desde la conquista española las alianzas de parentesco constituian un mecanismo de privilegio; la dictadura predominante en su historia republicana y la de los 35 últimos años no ha hecho sino reforzarlas, al convertirlas en fuente de poder dentro y fuera de la empresa. Para ascender de nivel en la organización, la persona debe tener algún parentesco -consanguíneo $o$ adquirido por matrimonio- con los propietarios.

i) Se ha encontrado una situación ambivalente en los principios morales dominantes cn el Paraguay. Por un lado, sus raíces guaranies le otorgan una base arquetipal que se manifiesta en una constante búsqueda de equilibrio entre las fuer- zas de la naturaieza y su misticismo explicativo de su acción; $y$ de otro lado, la base arquetipal española, de manifestaciones también religiosas, otorga valor a la propiedad y a la riqueza material, mientras que concibe al trabajo como un medio de expiación de pecados para alcanzar la salvación en una supuesta vida cterna.

Esto ha producido en los paraguayos un mestizaje de sus principios de vida, predominando la influencia española, que pudo imponerse principalmente a través del modelo económico, la religión y el idioma. Sin embargo, en la comunicación los paraguayos han logrado hacer prevalocer su idioma nativo, y si se considera que el habla es un medio por el cual los seres humanos transmiten un código de significado cuyas reglas se expresan, se forman o se simulan en determinados sentimientos, se comprenderá la trascendericia de este hecho.

j) La cultura de la empresa es resultadc del desarrollo social, económico, polítice y cultural $y$ de la dinámica arquetipal predominante en el contexto de la sociedad misma, y no $\mathrm{cl}$ simple cnunciado de valores y creencias divorciadas de las condicioncs que crean las formas de organizar. ción. El análisis de estos parámetros hé permitido inferir que la cultura $\mathrm{cn}$ la $\mathrm{cm}$. presa paraguaya se caractcriza por la autoridad paternalista, la ideología de organi. zacionesautoritarias y la mentalidad tribal todocllodentrode un modelo mecanicista Es decir, es una cultura de función, de tipe autoritaria, clánica.

k) Respecto del empleo de modelo: gerenciales, entre los directivos existe li falacia de pensar que la administración e: la racionalidad técnica y signilica la apli cación de modelos de gestión clásicos 
tradicionalmente conocidos como portadores de una lógica neutral y universal, es decir, aplicable a toda situación. En este sentido, la cultura es vista como un obstáculo al establecimiento de procedimientos formales, racionales, uniformes y unificantes. Este critcrio impide a los gerentes articular a la organización y a sus hombres teniendo en cuenta su realidad social particular y, a partir de ello, generar nuevos modelos que aprovechen las potencialidades propias de la sociedad y de la cultura particular en su contexto. La lógica de la eficiencia no alcanza a concretarse y se atribuye csta falla a los aspectos culturales.

\section{Referencias Bibliográficas}

BARTOLI, A, Communication et organisation: pour une politique générale cohérente. Paris, Editions d'organisation, 1990.

BERRY, M. el MATHEU, M., "Un regard d' ethnographe dans l'entreprise", Revue Françaisede Gestion, Paris, (58), 1985.

CONEXIONES, Número spécial sur la culture, (56), 1987.

CROZIER M. et FRIEDBERG E.. L'acteur et le sy.steme, Paris, Editions du Seuil,1977.

ERALY A., La structuration de lenterprise: la rationalité en action. Bruxelles, Editions de 1'Université de Bruxelles, 1988.

FROST, P.etal, Organizational cullure, Beverly Hills, California, Sage Publications, 1985.
1) Finalmente, se observa que el gerente cumple un rol esencial en la formación de la cultura de la cmpresa y en el intercambio entre ella y su cntorno social. Por ello, es necesario que comprenda cómo su propia cultura afecta su modo de pensar como individuo. Debe preguntarse lo que implican las transficrencias con el objetivo de hacer de la relación sociedad microsociedad. con él como nexo, una verdadera relación en beneficio de la empresa.
GAUTHEY F., RATIN I. et RODGERS I. Leaders sans frontieres, le défi des différences, Paris, Mac Graw Hill, 1988.

GAUTHEY F.et XARDEL.D.,Lemanagement interculturel, Presses universitaires de France, Que sais je, 1990.

GOGUELIN P., Le management psychologique des organisations, Paris. Entreprise Modeme d'edition, 1989.

HOFSTEDE G. Ot BOLINGER D., Les différences culturelles dans le management, Paris, Editions d' organisation, 1987.

INSTITUT DES SCIENCES DU TRAVAIL. "Culture d'entreprise, vous avez dit culture", Dossier, (12), 1990.

JUNG, Carl, Analyse psychologique de l'economie appliquée, Institut 
d'Administration et de Gestion, Universite Catholique de Louvain, 1990 1991.

LUHMANN, N., "A general theory of organized social systems", in Hofstede y Kassem (ed.), European contribution in organization theory. Amsterdam. 1982.

LUHMANNen SARUBIV., "Hacia una nueva epistemología de las Ciencias Sociales", Revista Paraguaya de Sociologia, Asunción, 26 (75): 73-106, may.- ago., 1989.

MARCH y SIMONS, Organisations, Dunod, 1974.

MICHAUD, G., Identités collectives et relations inter-culturelles. Ed. Complexe, 1978.

MINTZBERG, H., Le management, Paris, Editions d'organisation, 1990.

...,The nature of managerial work, New York. Harper y Row, 1973.
...., Le pouvoir dans les organisations, Paris, Editions d'organisation, 1986.

....The structure $d u$ organisations, Englewood Cliffs, N. J., Prentice Hall, 1979.

MORGAN, G.. Images of organisations. Beverly IIills, California, Sage Publications, 1988.

SHEIN E., Organizational culture and leadership, Jossey Bass, 1988.

STRATEGOR, Stratégie, structurc, décision, identité, Paris, Intereditions, 1989.

THEVENET, J., L'audit de la culture. Paris, Editions d'organization, 1986.

VACHETTE J.,"Peut-on mettre en évidence la culturedel'entreprise", Revue Francaise de Gestion, Paris, 1984. 